\title{
Lidocaine bolus may facilitate computed tomographic coronary angiography in patients with frequent premature ventricular contractions
}

\author{
Jerzy Pręgowski', Jan Jastrzębski', Cezary Kępka², Mariusz Kruk², Marcin Demkow², Łukasz Kalińczuk², \\ Rafał Wolny', Michał Ciszewski', llona Michałowska ${ }^{3}$, Adam Witkowski ${ }^{1}$ \\ ${ }^{1}$ Department of Cardiology and Invasive Angiology, Institute of Cardiology, Warsaw, Poland \\ 2Coronary Artery and Structural Heart Disease Department, Institute of Cardiology, Warsaw, Poland \\ 3Radiology Department, Institute of Cardiology, Warsaw, Poland
}

Postep Kardiol Inter 2013; 9, 3 (33): 199-204

DOI: 10.5114 /pwki.2013.37496

\begin{abstract}
Introduction: Heart rate irregularities are the major limitations of computed tomographic coronary angiography (CTCA) due to severe motion artifacts.

Aim: To evaluate the safety and efficacy of a lidocaine intravenous bolus in preserving good image quality by the transient suppression of premature ventricular contractions (PVC) during the CTCA scan.

Material and methods: The study group comprised 67 consecutive patients with sinus rhythm and numerous PVC scheduled for CTCA. Intravenous boluses of 25-50 mg lidocaine were given after calcium score assessment and immediately before CTCA. The control group comprised 67 patients with sinus rhythm without PVC matched according to the body mass index (BMI), age, sex, and calcium score. All coronary vessel segments were assessed for image quality and presence of significant stenosis.

Results: As compared with calcium score assessment, after administration of lidocaine and during the CTCA scan PVC were completely suppressed in $22(40 \%)$, reduced in $10(18 \%)$, unchanged in $18(32 \%)$, and intensified in $5(10 \%)$ patients. Overall, there were 32 (58\%) patients with sinus rhythm during CTCA as compared with only 11 (20\%) patients free from PVC during calcium score assessment $(p<0.001)$. Image quality in 871 coronary segments including both the study group and control patients was worse in patients with PVC $(p<0.0001)$. However, there was no statistically significant difference in the number of patients with at least one segment of non-diagnostic quality ( $6 \%$ vs. $12 \%, p=0.36$; respectively).

Conclusions: Single lidocaine bolus given prior to CTCA is safe, may temporarily eliminate or reduce the intensity of arrhythmia, and hence results in improved quality of CTCA in patients with numerous PVC.
\end{abstract}

Key words: computed tomography coronary angiography, artefacts, lidocaine.

\section{Introduction}

Computed tomographic coronary angiography (CTCA) is an established non-invasive tool allowing anatomical evaluation of coronary artery disease (CAD) [1]. However, motion artifacts caused by heart rate irregularities remain challenging for physicians (Figure 1); and in many centers arrhythmia is still a contraindication for CTCA [2, 3]. It is well known that lidocaine is very effective in suppressing premature ventricular contractions (PVC) $[4,5]$. After an intravenous bolus of the drug the therapeutic level is achieved very fast, but it also falls very rapidly as lidocaine is quickly metabolized $[3,4]$.

\section{Aim}

The aim of this study was to analyze retrospectively the safety and efficiency of a lidocaine bolus given before CTCA to improve CTCA quality by reducing the number of arrhythmia-induced artifacts.

\section{Material and methods}

The current study was designed as retrospective case controlled analyses based on the data from a large single center CTCA registry. The registry was approved by the local Ethic Committee. In our CT department we took the approach to use a lidocaine bolus in patients with numerous PVC who are referred for CTCA in order to temporarily suppress the arrhythmia. The study group comprised consecutive patients with sinus rhythm and numerous PVC who were scheduled for CTCA and in whom a lidocaine bolus was administered prior to CTCA examination. Patients with a history of prior coronary revascularization or known allergy to

Corresponding author:

Jerzy Pręgowski MD, Department of Cardiology and Invasive Angiology, Institute of Cardiology, 42 Alpejska St, 04-628 Warsaw, Poland, fax: +48 2234345 06, e-mail: jerzypregowski74@gmail.com

Received: 5.12.2012, accepted: 12.06.2013. 


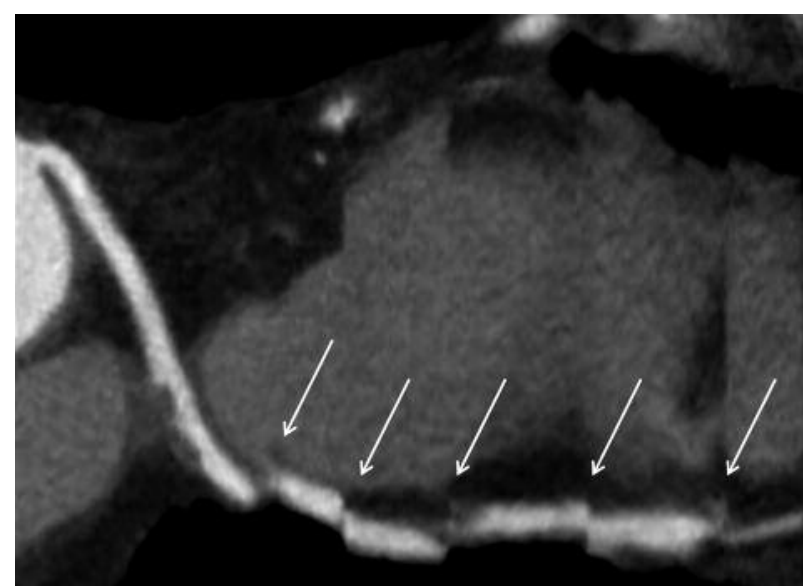

Fig. 1. Step-ladder artifacts (arrows) caused by numerous PVC

lidocaine were excluded. The control group comprised patients with sinus rhythm without any ectopic beats matched according to the body mass index (BMI), age, sex, and calcium score.

\section{Lidocaine administration and ECG tracing analysis}

All patients from the study group received lidocaine prior to CTCA examination. Intravenous boluses of $25 \mathrm{mg}$ to $50 \mathrm{mg}$ (up to a total dose of $100 \mathrm{mg}$ ) were given at the discretion of the physician supervising the examination, after calcium score assessment and immediately before CTCA. The arbitrary decision was made not to exceed the dose of $100 \mathrm{mg}$. The available ECG tracings recorded during calcium score and contrast CTCA were analyzed for the presence, number, and pattern of PVC.

\section{CTCA data acquisition and analyses}

The CTCA dataset was acquired with a dual-source 64slice CT scanner (Somatom Definition, Siemens, Forchheim, Germany). An ECG-gated helical retrospective acquisition protocol was performed with $330 \mathrm{~ms}$ rotation time, $0.6 \mathrm{~mm}$ collimation, and tube voltage of 100-140 kV adjusted manually for body mass index. The temporal resolution was $83 \mathrm{~ms}$ and the slice thickness $0.6 \mathrm{~mm}$ with an increment of $0.4 \mathrm{~mm}$. ECG-based tube current modulation was used in all patients with the acquisition window set at $40-70 \%$ of the R-R interval. If PVC occurred during acquisition, the tube current modulation was automatically off. Coronary wall calcification was assessed visually and determined quantitatively with the use of a standard builtin algorithm on an off-line workstation (Syngo Multimodality Workplace $^{\mathrm{TM}}$, Siemens). Peak arterial enhancement time was determined by the test bolus technique. During scan acquisitions, the contrast medium was injected at a rate of 5$6 \mathrm{ml} / \mathrm{s}$, followed by a $40-\mathrm{ml}$ saline flush from an automatic injector. In all patients nitrates were administered sublingually $(0.8 \mathrm{mg})$. In patients with a heart rate above $65 / \mathrm{min}$, intravenous metoprolol (sequential doses of $2.5 \mathrm{mg}$, maximal dose $15 \mathrm{mg}$ ) was given prior to the calcium score scan. Raw data at the phase of the R-R interval with the least motion was used to obtain the optimal reconstructions. The initial reconstruction was chosen automatically by a vendor provided algorithm (BestPhase; Siemens Healthcare) which interpolated the raw data at all available intervals and searched for the single phase of the RR cycle with the fewest artifacts. If clinicians were not satisfied with the automatically optimized phase reconstruction, additional phases were applied and, if necessary, manual ECG editing was performed at additional phases. All coronary vessel segments were assessed according to the modified AHA classification (model of coronary tree with 13 segments) [6] for image quality and presence of significant stenosis by an experienced reader and classified as follows: 1 - absence of artifacts; 2 - minor artifacts; 3 - considerable artifacts but maintained visualization of arterial lumen; 4 - diagnostically limited due to step-ladder artifacts; 5 - nondiagnostic due to severe motion artifacts or severe calcifications. The CTCA studies with an assigned score of 5 in any coronary artery were defined as non-diagnostic studies. The segments were scored for the presence of vessel stenosis using the following classification: 1 - without lesion; 2 - stenosis of 0-30\%; 3 - stenosis of $30-70 \%$; 4 - stenosis of $70-90 \%$; 5 - stenosis of > $90 \%$; 6 - nondiagnostic due to image quality. The radiation exposure for CTCA was quantified by using dose-length product (DLP) and the volume CT dose index (CTDI $\left.{ }_{\text {vol }}\right)$. These values were obtained from the scanner console. For radiation dose independent from the pitch the weighted $\mathrm{CT}$ dose index (CTDIw) was calculated for each patient.

\section{Statistical analysis}

Continuous data with normal distribution are presented as means with standard deviation while non-normally distributed variables are presented as medians with interquartile ranges (IQR). An independent samples $T$-test, paired $T$-test, Mann-Whitney test and Wilcoxon test were used as appropriate to assess differences between continuous variables. Categorical variables were compared with the $\chi^{2}$ test.

\section{Results}

\section{Patient population}

Between March 2009 and July 2011, we performed CTCA scans in 4800 patients. There were 79 consecutive patients with ventricular arrhythmias who were given an intravenous bolus of lidocaine prior to examination. We administered the lidocaine in increasing doses from $25 \mathrm{mg}$ to $100 \mathrm{mg}$ depending on the decision on achieved suppression of PVC. Eight patients received $25 \mathrm{mg}, 53$ patients received $50 \mathrm{mg}$, 3 patients $75 \mathrm{mg}$, and 15 subjects $100 \mathrm{mg}$ of lidocaine. We did not observe significant adverse effects of lidocaine administration except for transient tinnitus in a single patient and transient symptomatic sinus bradycardia (35/min) in 
Table 1. Demographics and CTCA procedural variables

\begin{tabular}{|c|c|c|c|}
\hline Variable & Study group $(n=67)$ & Control group $(n=67)$ & Value of $p$ \\
\hline Age, mean \pm SD [years] & $61.1 \pm 10.7$ & $61.3 \pm 10.5$ & \multirow[t]{2}{*}{0.90} \\
\hline Men, $n(\%)$ & $31(46.27)$ & $31(46.27)$ & \\
\hline Body mass index, mean $\pm \mathrm{SD}\left[\mathrm{kg} / \mathrm{m}^{2}\right]$ & $28.2 \pm 5.2$ & $28.2 \pm 5.1$ & 0.98 \\
\hline Ca score & $10.2($ IQR 0.0-137.6) & 6.6 (IQR 0.0-134.6) & 0.97 \\
\hline Tube potential of $100 \mathrm{kVp}, n(\%)$ & $17(28.8)$ & $9(18.4)$ & \multirow[t]{3}{*}{0.10} \\
\hline Tube potential of $120 \mathrm{kVp}, n(\%)$ & $42(71.2)$ & $38(77.5)$ & \\
\hline Tube potential of $140 \mathrm{kVp}, n(\%)$ & 0 & $2(4.1)$ & \\
\hline$\beta$-Blockers, $n$ (\%) [mg] & $40(59.7)$ & $27(40.3)$ & 0.04 \\
\hline 2.5 & $17(25.4)$ & $8(11.9)$ & \multirow[t]{5}{*}{0.42} \\
\hline 5 & $17(25.4)$ & $10(14.9)$ & \\
\hline 7.5 & $2(3.0)$ & $2(3.0)$ & \\
\hline 10 & $3(4.5)$ & $7(10.4)$ & \\
\hline 15 & $1(1.5)$ & 0 & \\
\hline Mean heart rate during Ca scan, mean \pm SD & $68.2 \pm 13.7$ & $64.1 \pm 10.1$ & 0.06 \\
\hline Mean heart rate during CTCA, mean \pm SD & $62.8 \pm 8.9$ & $60.8 \pm 8.8$ & 0.20 \\
\hline Dose-length product [mGy $\times \mathrm{cm}]$ & 1058.5 (IQR 810.0-1295.5) & 903.5 (IQR 609.0-1105.0) & 0.01 \\
\hline CTDIvol & $105.40($ IQR 87.3-127.7) & $98.2($ IQR 80.3-114.9) & 0.18 \\
\hline Pitch, mean \pm SD & $0.27 \pm 0.15$ & $0.26 \pm 0.03$ & 0.04 \\
\hline Scan time, mean \pm SD $[\mathrm{s}]$ & $10.6 \pm 2.0$ & $9.9 \pm 2.3$ & 0.09 \\
\hline
\end{tabular}

Ca score - calcium score, $C T D I_{\text {vol }}$-volume computed tomography dose index, CTCA - computed tomographic coronary angiography

a single patient who received both $\beta$-blocker and lidocaine. The bradycardia was successfully treated with atropine administration. It is unclear if bradycardia was related to $\beta$-blocker or lidocaine administration.

In 12 cases the contrast-enhanced CTCA examination was abandoned after calcium score assessment. In one case the reason was the aforementioned bradycardia and in 11 patients, in the arbitrary opinion of the physician supervising the examination, lidocaine was not effective enough in the suppression of PVC; and the risk of obtaining non-diagnostic images was too high. These 12 patients were referred for invasive angiography. Complete scan protocol (calcium scoring and CTCA) was performed in $67 \mathrm{pa}$ - tients for which the control group was matched. Baseline patients' characteristics are presented in Table 1.

\section{Lidocaine effect}

Out of 67 patients paired ECG data obtained during calcium scoring (prior to lidocaine examination) versus during CTCA scan were available for 55 patients (Figure 2). Premature ventricular contractions during calcium score assessment were present in 44 patients (80\%) and included: numerous single ventricular extrasystoles (39 patients), ventricular bigeminy (3 patients), couplets (1 patient), and nonsustained ventricular tachycardia composed of 3 beats (1 patient). In 11 patients no PVC occurred during calcium

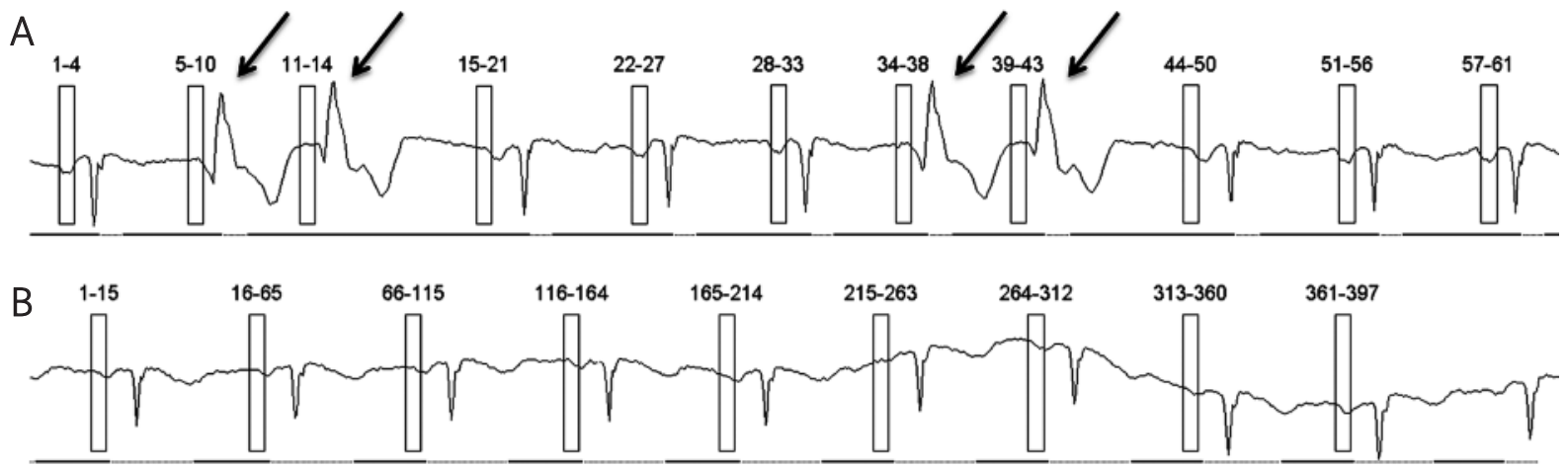

Fig. 2. ECG tracing with 4 PVC during calcium score examination (A) and without PVC during contrast-enhanced CTCA after administration (B) 
Table 2. Impact of the lidocaine bolus given immediately before CTCA on the heart rate

\begin{tabular}{lccc}
\multicolumn{1}{l}{$\begin{array}{l}\text { Variable } \\
\text { Mean heart rate }\end{array}$} & $\begin{array}{c}\text { CTCA scan } \\
\text { (after lidocaine bolus) }\end{array}$ & Value of $p$ \\
\hline \begin{tabular}{l} 
Ventricular extrasystoles \\
\cline { 2 - 3 } Single ventricular extrasystoles
\end{tabular} & $68.2 \pm 13.7$ & $62.9 \pm 8.9$ & 0.0004 \\
\hline $\begin{array}{l}\text { Ventricular bigeminy } \\
\text { Couaplets }\end{array}$ & $39 / 55(80.0 \%)$ & $23 / 55(42.0 \%)$ & 0.002 \\
\hline Ventricular tachycardia & $3 / 55(5.4 \%)$ & $22 / 55(42.1 \%)$ & 0.002 \\
\hline No. of contractions during image acquisition & $1 / 55(1.8 \%)$ & $0 / 55(0 \%)$ & $1 / 55(1.7 \%)$ \\
\hline
\end{tabular}

score acquisition, but significant arrhythmia was observed immediately before and after this scan. As compared with calcium score assessment, after administration of lidocaine and during the CTCA scan PVC were completely suppressed in 22 patients (40\%), in 10 (18\%) patients the number of ectopic ventricular beats per scan was reduced, in 18 (32\%) patients there was no reduction in arrhythmia intensity, and in 5 (10\%) patients the arrhythmia was intensified (Table 2). Overall, there were $32 / 55$ patients (58\%) with absolutely normal sinus rhythm during CTCA as compared with only $11 / 55$ patients (20\%) free from ectopic beats during calcium score assessment $(p<0.001)$.

Additional analysis was performed for the broader group comprising also 12 patients in whom CTCA was abandoned even though they received lidocaine. If the assumption was made that PVC were present in those 12 subjects both during calcium score examination and during contrastenhanced CTCA (if it was performed) the effect of lidocaine was still significant (normal sinus rhythm during contrast scan in $32 / 67$ patients (48\%) vs. $11 / 67$ (16\%) during calcium score before lidocaine $(p<0.001))$.

\section{Procedural variables}

The heart rate during calcium score assessment tended to be higher in the study group $(p=0.06)$, but there was no difference in heart rate during contrast examination $(p=0.2)$. $\beta$-Blockers were used more frequently in the study group, but the dose used was similar in the two groups (Table 1). The tube potential in both arms and scan times were similar, but the dose-length product was larger in patients with ventricular arrhythmia $(p=0.01)$ (Table 1). This could be explained by lack of tube current modulation during CTCA after an ectopic ventricular beat.

\section{Per segment image analyses and quality}

Median calcium score was similar in the study group (10.2 IQR 0.0-136.6) and in the control patients (6.6 IQR 0.0134.6) $(p=1.0)$. Ninety-eight segments were excluded from the analysis of the disease extent by CTCA because of low image quality (43 segments) or variations in vessel anatomy or small artery diameter (55 segments). The overall sever- ity of coronary lesions was similar in the study group versus control patients (Table 3). There were 13 (19.4\%) patients with at least one segment with a $>70 \%$ diameter stenosis in the study group versus 15 (22.4\%) control patients $(p=0.8)$.

Image quality was assessed for 871 coronary segments in the study group and control patients. Overall, the quality was worse in patients with ventricular arrhythmias $(p<0.0001)$ (Table 3). Thirty-two (3.7\%) segments in the study population and 10 segments (1.1\%) in the control group were non-diagnostic due to severe motion artifacts or calcifications ( $p=0.001)$. Within the study group the number of motion artifacts in patients with complete PVC suppression following lidocaine administration was lower as compared to the poorer responders $(p=0.002)$ (Table 4).

\section{Per patient image quality analyses}

The analysis including 12 patients in whom CTCA was not performed because of lidocaine ineffectiveness showed that the percentage of patients with either at least a single non-diagnostic segment or non-responsive to lidocaine was higher than the proportion of patients with a non-diagnostic segment in the control group (20/79 (25\%) vs. 4/67 $(6 \%) ; p=0.004)$. However, if those 12 patients in whom contrast-enhanced CTCA was abandoned were excluded, we found no statistically significant difference in the number of patients with at least a single segment of non-diagnostic quality: $8(12 \%)$ vs. $4(6 \%)(p=0.36)$ (for study group and control patients, respectively).

\section{Discussion}

The main finding of the current study was that a single lidocaine bolus given immediately prior to CTCA was safe, temporarily eliminated or reduced the intensity of arrhythmia and facilitated CTCA in a substantial proportion of patients with numerous PVC referred for CTCA. The current manuscript although retrospective is the first report on the novel approach of temporal heart rhythm stabilization prior to CTCA.

Extensive calcifications and motion artifacts related to heart rhythm irregularities are major limitations of CTCA 
Table 3. Results of image quality and severity of coronary artery disease assessment in per-segment and perpatient analyses

\begin{tabular}{|c|c|c|c|}
\hline Variable & Study group $(n=67)$ & Control group $(n=67)$ & Value of $p$ \\
\hline Mean quality, mean \pm SD & $1.32 \pm 0.69$ & $1.15 \pm 0.41$ & 0.15 \\
\hline Patients with $\geq 1$ non-diagnostic segment, $n(\%)$ & $8(11.9 \%)$ & $4(6.0 \%)$ & 0.36 \\
\hline \multicolumn{4}{|l|}{ Image quality per segment } \\
\hline Absence of artifacts & $752 / 871(86.3 \%)$ & $808 / 871(92.8 \%)$ & \multirow[t]{5}{*}{$<0.0001$} \\
\hline Minor artifacts & $41 / 871(4.7 \%)$ & $29 / 871(3.3 \%)$ & \\
\hline Considerable artifacts but maintained visualization of arterial lumen & $31 / 871(3.6 \%)$ & $14 / 871(1.6 \%)$ & \\
\hline Diagnostically limited due to step-ladder artifacts & $15 / 871(1.7 \%)$ & 10/871 (1.1\%) & \\
\hline Non-diagnostic due to severe motion artifacts or severe calcifications & $32 / 871(3.7 \%)$ & 10/871 (1.1\%) & \\
\hline \multicolumn{4}{|l|}{ Disease extent per segment } \\
\hline Without lesion & $485 / 871(55.7 \%)$ & $560 / 871(64.3 \%)$ & \multirow[t]{7}{*}{0.20} \\
\hline $0-30 \%$ & $229 / 871(26.3 \%)$ & $172 / 871(19.7 \%)$ & \\
\hline $30-70 \%$ & $68 / 871(7.8 \%)$ & $70 / 871(8.0 \%)$ & \\
\hline $70-90 \%$ & $14 / 871(1.6 \%)$ & $34 / 871(3.9 \%)$ & \\
\hline$>90 \%$ & $4 / 871(0.5 \%)$ & $8 / 871(0.9 \%)$ & \\
\hline Nondiagnostic due to image quality & $32 / 871(3.7 \%)$ & $11 / 871(1.3 \%)$ & \\
\hline Nondiagnostic due to vessel anatomy & $39 / 871(4.5 \%)$ & $16 / 871(1.8 \%)$ & \\
\hline
\end{tabular}

Table 4. Comparison of image quality and severity of coronary artery disease in patients in whom PVCs were completely suppressed after lidocaine administration versus those with persistent PVCs

\begin{tabular}{|c|c|c|c|}
\hline Variable & $\begin{array}{l}\text { Patients with } \\
\text { suppressed PVCs } \\
\quad(n=41)\end{array}$ & $\begin{array}{l}\text { Patients with no } \\
\text { reduction in arrhythmia } \\
\qquad(n=26)\end{array}$ & Significance \\
\hline Mean quality & $1.31 \pm 0.62$ & $1.51 \pm 0.90$ & 0.13 \\
\hline Patients with $\geq 1$ non-diagnostic segment & $5(12.2 \%)$ & $3(11.5 \%)$ & 0.96 \\
\hline \multicolumn{4}{|l|}{ Image quality per segment } \\
\hline Absence of artifacts & $479 / 533(89.9 \%)$ & $273 / 338(80.8 \%)$ & \multirow[t]{5}{*}{0.0002} \\
\hline Minor artifacts & $19 / 533(3.6 \%)$ & $22 / 338(6.5 \%)$ & \\
\hline Considerable artifacts but maintained visualization of arterial lumen & $10 / 533(2.4 \%)$ & $21 / 338(6.2 \%)$ & \\
\hline Diagnostically limited due to step-ladder artifacts & $11 / 533(1.9 \%)$ & $4 / 338(1.2 \%)$ & \\
\hline Nondiagnostic due to severe motion artifacts or severe calcifications & $14 / 533(2.6 \%)$ & $18 / 338(5.3 \%)$ & \\
\hline Patients with $\geq 70 \%$ stenosis in $\geq 1$ segment & $10(24.4 \%)$ & $3(11.5 \%)$ & 0.20 \\
\hline
\end{tabular}

[7-9]. It is relatively easy to slow down the heart rate in patients with sinus rhythm with $\beta$-blockers and hence improve image quality [10-12]. However, $\beta$-blockers are not that effective in temporal suppression of arrhythmias such as frequent PVC $[13,14]$. Premature ventricular beats may be a manifestation of obstructive coronary artery disease [15] and occur in up to $10 \%$ of patients referred for CTCA $[16,17]$. In our experience the percentage of patients with PVC scheduled for CTCA is lower, most likely because of the awareness of the physicians who refer these subjects directly for invasive angiography. Recently it has been shown that if PVC occur during the calcium score scan, they are also present in the same number during subsequent CTCA and significantly worsen image quality [16]. Therefore, the presence of frequent PVC observed immediately before CTCA is considered as a relative contraindication for the procedure. Our results document that a lidocaine bolus given immediately after calcium score scanning and before CTCA effectively abolishes or reduces the number of PVC in a substantial proportion of patients. Therefore, this strategy may broaden the population of patients who may benefit from non-invasive anatomical assessment of coronary artery disease. However, it should be stated that lidocaine did not prove to be an ideal tool as in around $50 \%$ of patients the arrhythmia did not diminish after lidocaine and CTCA was either not performed or significant motion artifacts impaired image quality.

A recent study investigated a new algorithm for advanced arrhythmia rejection/compensation that allowed a decrease in radiation exposure with similar image qual- 
ity as compared with classical retrospectively ECG-gated helical scanning [18]. The combination of our pharmacological approach to patients with PVC and newer acquisition algorithms and techniques $[18,19]$ may be an optimal strategy to achieve high diagnostic accuracy CTCA studies among patients with various heart rates and rhythms.

The most frequent adverse effects of intravenous lidocaine administration are in general dose dependent, occur during continuous infusion or after a bolus given too quickly, and are related to central nervous system involvement $[5,20]$. In our study we observed only one transient episode of tinnitus and therefore our strategy seems to be safe. We observed that patients with PVC during calcium score scan received significantly higher radiation doses during CTCA compared to patients with normal sinus rhythm despite a favorable lidocaine effect. These findings seem to be in line with the data published so far [9, 21].

The major limitations of the study are its retrospective nature, paired ECG tracing from calcium score scan and CTCA scan were available only for $82 \%$ of the study group, and there was no routine invasive coronary angiography to verify the CTCA diagnostic accuracy. In addition, ideally each patient would have served as his own control with CTCA performed before and after lidocaine administration. Not all patients with PVC who were referred for CTCA were given lidocaine as our approach to this method was evolving in time.

In conclusion, a single lidocaine bolus given prior to CTCA is safe, may temporarily eliminate or reduce the intensity of arrhythmia, and hence may facilitate CTCA in a substantial proportion of patients with numerous PVC. Further prospective study is warranted to directly address the usefulness of lidocaine prior to CTCA in patients with numerous PVC.

\section{References}

1. Schroeder S, Achenbach S, Bengel F, et al. Cardiac computed tomography: indications, applications, limitations, and training requirements: report of a Writing Group deployed by the Working Group Nuclear Cardiology and Cardiac CT of the European Society of Cardiology and the European Council of Nuclear Cardiology. Eur Heart J 2008; 29: 531-556.

2. Mark DB, Berman DS, Budoff MJ, et al. ACCF/ACR/AHA/NASCI/ SAIP/SCAI/SCCT 2010 expert consensus document on coronary computed tomographic angiography: a report of the American College of Cardiology Foundation Task Force on Expert Consensus Documents. J Am Coll Cardiol 2010; 55: 2663-2699.

3. Tsiflikas I, Drosch T, Brodoefel H, et al. Diagnostic accuracy and image quality of cardiac dual-source computed tomography in patients with arrhythmia. Int J Cardiol 2010; 143: 79-85.

4. Collinsworth KA, Kalman SM, Harrison DC. The clinical pharmacology of lidocaine as an antiarrhythymic drug. Circulation 1974; 50: 1217-1230.

5. The clinical pharmacology of lidocaine as an antiarrhythmic drug. Medical Staff Conference, University of California, San Francisco. West J Med 1976; 124: 36-43.

6. Austen WG, Edwards JE, Frye RL, et al. A reporting system on patients evaluated for coronary artery disease. Report of the Ad Hoc Com mittee for Grading of Coronary Artery Disease, Council on Cardiovascular Surgery, American Heart Association. Circulation 1975; 51: 5-40.

7. Alkadhi H, Scheffel H, Desbiolles L, et al. Dual-source computed tomography coronary angiography: influence of obesity, calcium load, and heart rate on diagnostic accuracy. Eur Heart J 2008; 29: 766-776.

8. Brodoefel H, Burgstahler C, Tsiflikas I, et al. Dual-source CT: effect of heart rate, heart rate variability, and calcification on image quality and diagnostic accuracy. Radiology 2008; 247: 346-355.

9. Weustink AC, Neefjes LA, Kyrzopoulos S, et al. Impact of heart rate frequency and variability on radiation exposure, image quality, and diagnostic performance in dual-source spiral CT coronary angiography. Radiology 2009; 253: 672-680.

10. Mahabadi AA, Achenbach S, Burgstahler C, et al. Safety, efficacy, and indications of beta-adrenergic receptor blockade to reduce heart rate prior to coronary CT angiography. Radiology 2010; 257: 614-623.

11. Ropers U, Ropers D, Pflederer T, et al. Influence of heart rate on the diagnostic accuracy of dual-source computed tomography coronary angiography. J Am Coll Cardiol 2007; 50: 2393-2398.

12. Ferencik $\mathrm{M}$, Nomura $\mathrm{CH}$, Maurovich-Horvat $\mathrm{P}$, et al. Quantitative parameters of image quality in 64-slice computed tomography angiography of the coronary arteries. Eur J Radiol 2006; 57: 373-379.

13. Pitzalis MV, Mastropasqua F, Massari F, et al. Dependency of premature ventricular contractions on heart rate. Am Heart J 1997; 133: 153-161.

14. Pitzalis MV, Mastropasqua F, Massari F, et al. Holter-guided identification of premature ventricular contractions susceptible to suppression by beta-blockers. Am Heart J 1996; 131: 508-515.

15. Massing MW, Simpson RJ Jr, Rautaharju PM, et al. Usefulness of ventricular premature complexes to predict coronary heart disease events and mortality (from the Atherosclerosis Risk In Communities cohort). Am J Cardiol 2006; 98: 1609-1612.

16. Eicher W, Kau T, Niedermayer M, et al. Impact of PVCs noted during coronary calcium scan on image quality and accuracy in subsequent coronary dual-source CT angiography. Int J Cardiovasc Imaging 2011; 27: 601-610.

17. Matsutani H, Sano T, Kondo T, et al. ECG-edit function in multidetector-row computed tomography coronary arteriography for patients with arrhythmias. Circ J 2008; 72: 1071-1078.

18. Lee AM, Engel LC, Shah B, et al. Coronary computed tomography angiography during arrhythmia: Radiation dose reduction with prospectively ECG-triggered axial and retrospectively ECG-gated helical 128-slice dual-source CT. J Cardiovasc Comput Tomogr 2012; 6: 172-183.

19. Uehara M, Takaoka H, Kobayashi Y, et al. Diagnostic accuracy of 320-slice computed-tomography for detection of significant coronary artery stenosis in patients with various heart rates and heart rhythms compared with conventional coronary-angiography. Int J Cardiol 2012; doi:10.1016/j.ijcard.2012.02.017

20. Gil-Gouveia R, Goadsby PJ. Neuropsychiatric side-effects of lidocaine: examples from the treatment of headache and a review. Cephalalgia 2009; 29: 496-508.

21. Techasith T, Ghoshhajra BB, Truong QA, et al. The effect of heart rhythm on patient radiation dose with dual-source cardiac computed tomography. J Cardiovasc Comput Tomogr 2011; 5: 255-263. 